\title{
Chronische Morphintherapie und respiratorische Insuffizienz im Schlaf
}

\author{
Long-Term Opioid Therapy and Respiratory Insufficiency During Sleep
}

Autoren

Institute
J. E. S. Nolte ${ }^{1}$, F. Dette ${ }^{2}$, W. Cassel ${ }^{1}$, C. Riese ${ }^{1}$, M. Augsten ${ }^{1}$, U. Koehler ${ }^{1}$

Klinik für Innere Medizin, SP Pneumologie, Intensiv- und Schlafmedizin (Direktor: Prof. Dr. C. Vogelmeier),

Philipps-Universität Marburg

2 Klinik für Anästhesie und Intensivtherapie (Direktor: Prof. Dr. H. Wulf), Philipps-Universität Marburg eingereicht 22.10.2009 akzeptiert nach Revision 21. 1.2010

\section{Bibliografie}

DOI http://dx.doi.org/ 10.1055/s-0029-1243965 Pneumologie 2010; 64: 241-245 @ Georg Thieme Verlag KG Stuttgart · New York ISSN 0934-8387

\section{Korrespondenzadresse} Johannes E. S. Nolte

Klinik für Innere Medizin SP Pneumologie, Intensiv- und Schlafmedizin Universitätsklinikum Gießen und Marburg GmbH Standort Marburg Baldingerstraße 1 35033 Marburg johannesnolte@gmail.com

\section{Zusammenfassung \\ $\nabla$}

Ein zunehmender Anteil von Patienten mit chronischen Schmerzen wird mittels Morphingabe analgetisch behandelt. Es gibt Hinweise dafür, dass eine Hypersomnieneigung am Tage bei diesen Patienten vor allem durch Atmungsstörungen im Schlaf bedingt ist. Am Beispiel von drei Patienten unter Langzeitopioidtherapie werden die Polysomnografien vor und unter nächtlicher Beatmungstherapie dargestellt. Als auffällige Befunde konnten zentrale Atmungsstörungen, einhergehend mit vermindertem Tief- und REM-Schlaf, objektiviert werden. Unter der eingeleiteten nicht invasiven Beatmungstherapie fand sich eine deutliche Verbesserung des respiratorischen Status mit Zunahme des Tiefschlafs und moderater Abnahme der Hypersomnie. Bei Patienten mit chronischer Opioidtherapie und Hypersomnie sollte an das Vorliegen zentraler Atmungsstörungen gedacht werden.

\section{Einleitung}

$\nabla$

Bei der Therapie chronischer Schmerzen ist, entsprechend der Leitlinie, dann ein Opioid zu geben, wenn im Rahmen der Stufentherapie eine ausreichende Schmerzbefreiung mit einem peripheren Analgetikum nicht zu erzielen ist. Aus der klinischen Erfahrung und der wissenschaftlichen Literatur ist bekannt, dass Patienten mit längerfristigem Opioidgebrauch unter anderem über Müdigkeit und Konzentrationsschwierigkeiten sowie allgemeine Beeinträchtigungen der psychischen Leistungsfähigkeit klagen [1]. Sowohl Art und Ausprägung als auch die Ätiologie dieser Beschwerden sind unklar. Es gibt Hinweise dafür, dass eine Hypersomnie am Tage bei Patienten mit chronischer Opioidtherapie vor allem durch nächtliche Atmungsstörungen bedingt ist.

\section{Abstract \\ $\nabla$}

An increasing proportion of the patients with chronic pain are being treated with opioids on a long-term basis. There are indications that the causes of hypersomnia in patients under chronic opioid therapy are primarily related to breathing disorders during sleep. Hence, we compared the polysomnographies of three hypersomnic patients receiving long-term opioid therapy before and during nocturnal non-invasive ventilatory therapy. Significant findings were a central breathing pattern accompanied by reduced deep and REM sleep. On applying non-invasive ventilatory therapy, there was a significant improvement of respiratory status with an increase of deep sleep as well as a moderate decrease in hypersomnia. In patients under chronic opioid therapy with hypersomnia, the presence of central breathing disorders should be considered.

\section{Atmungsregulation}

Der Atemrhythmus wird durch ein neuronales Netzwerk respiratorischer Neurone in der medulla oblongata (formatio reticularis) und der Pons generiert [2]. Über efferente Nervenbahnen werden dann über den so genannten Rhythmusgenerator verschiedene Anteile des Atemapparates innerviert: Atemmuskulatur, extra- und intrapulmonale Atemwege, Lungengefäße und periphere Chemorezeptoren. Die Aktivierung des neuronalen Netzwerks erfolgt über Antriebsmechanismen, die sowohl aus der Körperperipherie als auch von übergeordneten Hirnregionen kommen. Das Netzwerk wird stimuliert oder gebremst durch Afferenzen, zu denen die chemischen (Hypoxie, Hyperkapnie, Azidose) sowie die verhaltens- und wachheitsabhängigen Atmungsantriebe (orexinerges System) gehören. Schädigungen des Hirnstamms können das neuronale respirato- 
rische Netzwerk beeinträchtigen und konsekutiv zum Auftreten von Atmungsstörungen führen. Der chemische Atmungsantrieb wird in Abhängigkeit von Sauerstoff- und Kohlendioxidpartialdruck sowie vom ph-Wert des arteriellen Blutes und des Liquor cerebrospinalis geregelt. Periphere Chemorezeptoren in den Glomera carotica sowie die im Hirnstamm lokalisierten zentralen Chemorezeptoren fungieren als Sensoren, die den Rhythmusgenerator antreiben oder hemmen. Ist der $\mathrm{CO}_{2}$-empfindliche Atmungsantrieb gestört, kann dies ausgeprägte Hyoventilationen und zentrale Apnoen zur Folge haben. Mit dem Einschlafen entfallen die Wachheitsantriebe der Atmung, es kommt zudem zu einer Abschwächung des chemischen Atmungsantriebs mit diskretem Anstieg des $\mathrm{CO}_{2}$-Partialdrucks im Schlaf.

\section{Opioide und Schlaf \\ $\nabla$}

Unter „Opioiden“ werden sowohl nativ vorkommende (i.e. Opiate) als auch synthetisierte Substanzen mit morphinartiger Wirkung subsumiert. Die Entdeckung spezifischer Opioidrezeptoren in den 70er-Jahren des vergangenen Jahrhunderts im Tiermodell [3-6] und die folgende Anwendung am Menschen [7] war ein Meilenstein in der spinal wirkenden und systemischen Anästhesie. Opioide binden reversibel an Opioidrezeptoren, von denen mindestens drei Haupttypen zu unterscheiden sind: $\mu-, \mathrm{K}-$ und $\delta$-Rezeptoren. Diese sind weit verbreitet im Gehirn, Rückenmark sowie in der Nähe peripherer Nozizeptoren zu finden [8].

Opioidrezeptoren befinden sich auch in den bei der Schlafregulation aktiven Nuklei [9]. Opioiden wird eine Beteiligung bei der Einleitung und Aufrechterhaltung des Schlafes zugesprochen [10]. Im Weiteren konnte in einigen Studien gezeigt werden, dass die Wachzeit innerhalb der nächtlichen Ruhephase erhöht wird, die totale Schlafzeit, Schlafeffizienz, Delta-Schlaf und REMSchlaf reziprok dazu erniedrigt [11-13]. Durch die direkte Verbindung von Schmerzbahnen zu den Schlafzentren im Hirnstamm kann ein Weckreiz und damit eine Schlafunterbrechung noch vor bewusstem Wahrnehmen von Schmerz erfolgen.

Dimsdale konnte nachweisen, dass eine einmalige niedrigdosierte Opioidgabe bei gesunden Probanden bereits eine beeindruckende Erniedrigung des Tiefschlafes um 30-50\% zur Folge hat, das Schlafstadium 2 im Ausgleich dafür aber steigt. Eine Reduktion des REM-Schlafes oder eine Erhöhung des Apnoe-Hypopnoe-Index oder der Anzahl von Arousals im Schlaf konnte bei einmaliger Gabe nicht beobachtet werden [14].

\section{Opioide und Atemdepression}

$\nabla$

Die gefürchtetste Nebenwirkung einer Opioid-Dauertherapie ist die Atemdepression. Über zentrale $\mu_{2}$-Rezeptoren wirken die Opioide über eine Verringerung der $\mathrm{CO}_{2}$-Empfindlichkeit des Atemzentrums atemdepressiv. Bei Patienten mit dauerhafter Erhöhung des $\mathrm{paCO}_{2}$ büßt das Atemzentrum an $\mathrm{CO}_{2}$-Sensitivität [15] ein und die Hypoxie bestimmt den Atemantrieb [16]. Die Ausprägung der Atemdepression ist direkt proportional zur analgetischen Potenz des Opioids. Als leichte Verlaufsform kann die Atemdepression in Form von Hypoventilationen und verminderter Atemfrequenz auftreten. In diesem Stadium können die Patienten der Aufforderung zum aktiven Atmen noch nachkommen („Kommandoatmung“). Bei höherer Dosierung kommt es zu einer unregelmäßigen periodischen Atmung mit Apnoen bis hin zum kompletten Atemstillstand. Eine periodische Atmung, auch
Biot-Atmung genannt, wird primär bei Erkrankungen des zentralen Nervensystems wie Meningitis, Apoplexie und zerebraler Blutung beschrieben. Ebenso ist eine periodische Atmung bei Patienten mit Lungenödem unter extremer Höhe bekannt. Bei Patienten mit schmerzbedingter Morphinapplikation ist eine klinisch relevante Atemdepression deutlich geringer ausgeprägt. Die Toleranzentwicklung bei Opioiden kann zu einer Verkürzung von Wirkdauer und -effizienz führen.

Das aktivierende retikuläre System (ARS) erfährt als übergeordneter Taktgeber für die Atemregulation eine zusätzliche Dämpfung durch die Opioide. Die Vigilanz (Wachheit) hat eine elementare Bedeutung für die Atemregulation. Erst eine ausreichend hohe Vigilanz ermöglicht eine adäquate Reaktion auf einen Reiz wie Hyperkapnie oder Hypoxie. Sind die Rezeptoren im retikulären System durch Opioide besetzt, so ist der übergeordnete Kontrollmechanismus gestört. Die zusätzliche Gabe von Sedativa und Antidepressiva hat eine weitere Zunahme dämpfender Einflüsse auf die Vigilanz zur Folge. Hinsichtlich der Beurteilung einer opioidbedingten Atemdepression sind somit neben direkten Effekten auf den Atemrhythmusgenerator auch zentral induzierte sedative Wirkungen von großer Bedeutung.

\section{Opioide und schlafbezogene Atmungsstörungen 7}

Bislang existieren nur vereinzelte Untersuchungen zur nächtlichen Atmung unter Opioidtherapie beim Menschen [2,17-19]. Die gefundenen Atmungsstörungen waren primär zentraler Genese und unterschieden sich grundlegend von denen bei Herzinsuffizienz, welche durch eine crescendo-decrescendo-Atmung („Cheyne-Stokes-Atmung“) charakterisiert sind. Die akute Applikation von Opioiden führt zu respiratorischen Pausen, Verzögerungen der Exspiration sowie periodischer Atmung bei normalem oder reduziertem Tidalvolumen. Das periodische Atemmuster imponiert mitunter wie die Unregelmäßigkeit des Herzrhythmus bei einer Arrhythmia absoluta.

In einer Zusammenstellung der Patientendaten von 18 zwischen 1966 und 2005 durchgeführten Studien zu den Themen Opioide - Schlaf - Atmung - Tagesbefindlichkeit kommt Wang zu dem Ergebnis, dass aufgrund mangelnder methodischer Vergleichbarkeit und geringen Fallzahlen jedoch nur eingeschränkte Aussagen über die pathophysiologischen Interaktionen möglich sind [19]. Walker u. Mitarb. untersuchten retrospektiv 60 Patienten mit chronischer Opioidtherapie (Therapiedauer länger als 6 Monate) bei überwiegend nicht maligner Grunderkrankung im Hinblick auf das Auftreten von schlafbezogenen Atmungsstörungen und verglichen deren Daten mit denen eines gesunden Kontrollkollektivs [20]. Patienten mit Opioidtherapie wiesen signifikant häufiger zentrale Atmungsstörungen (12,8 vs $2,1 \mathrm{~h}$ ) auf, die vorwiegend im NREM-Schlaf zu objektivieren waren. Mit zunehmender Morphindosis stieg auch die Häufigkeit der Atmungsstörungen. Das Morphindosisäquivalent betrug im Mittel 143,9 mg (Bereich 7,5 bis $750 \mathrm{mg}$ ). Bei $92 \%$ der Patienten mit einem Morphindosisäquivalent von > $200 \mathrm{mg}$ konnten zentrale Atmungsstörungen nachgewiesen werden, bei lediglich $61 \%$ war dies bei unter $200 \mathrm{mg}$ der Fall. Im Kontrollkollektiv hatten nur 5\% der Patienten zentrale Atmungsstörungen.

Problematisch bei der Betrachtung der Atemdepression erscheint die nicht vorhandene Definition der Atemdepression im Rahmen der intrathekalen Morphinapplikation. In einer Übersichtsarbeit von Ko wird festgestellt, dass 46\% der ausgewerteten Publikationen das Wort „respiratory depression“ (RD) undefiniert benut- 

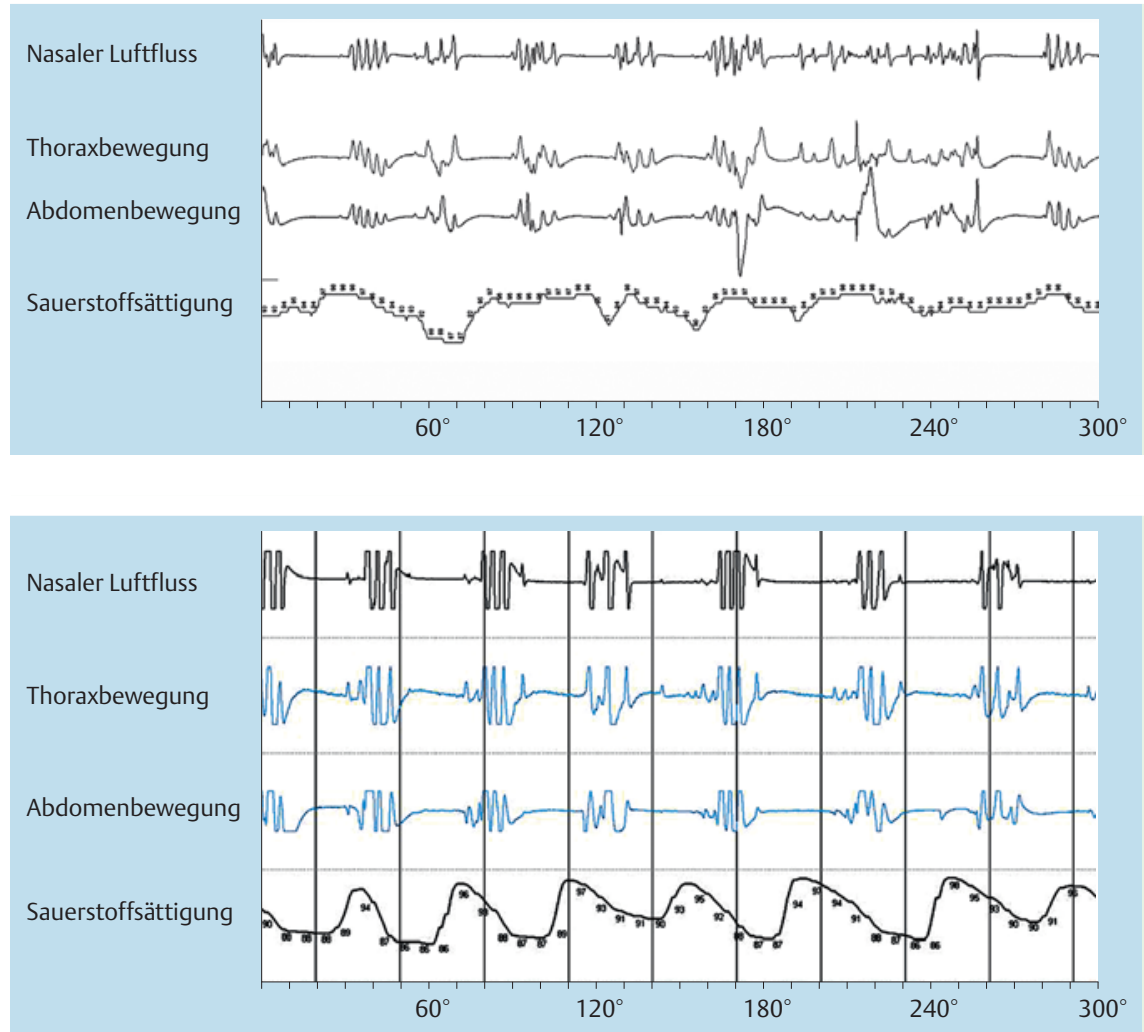

Abb. 1 Patient 1; 5-Minuten-Segment der Atmungsparameter aus der diagnostischen Polysomnografie.

Abb. 2 Patient Nr. 2; 5-Minuten-Segment der Atmungsparameter aus der diagnostischen Polysomnografie. zen [21]. 25\% definierten die RD alleinig über die erniedrigte „respiratory rate“ (RR) und lediglich $29 \%$ der Studien konkretisierten die RD mit mehr als nur der RR. Hier wurden beispielsweise additiv erhoben: hohe arterielle $\mathrm{pCO}_{2}$-Werte, niedrige $\mathrm{SpO}_{2}$, erhöhtes Sedationsniveau (LOS), erniedrigte Atemantwort auf Hyperkapnie und Hypoxie, die nötige Naloxon-Antagonisierung oder eine Kombination dieser Parameter. Somit fehlt eine einheitliche Definition der Atemdepression bei Opioidapplikation, was eine Erhebung ihrer Inzidenz sehr erschwert.

\section{Fallberichte}

Nächtliche Atmungsstörungen bei Patienten unter chronischer Opioidtherapie sollen beispielhaft anhand von drei Fallberichten dargestellt werden:

Fall 1: Es handelt sich um einen 37-jährigen Patienten (Gewicht $109 \mathrm{~kg}$, Größe $178 \mathrm{~cm}$, BMI $35 \mathrm{~kg} / \mathrm{m}^{2}$ ), der sich im Zentrum für Schlafmedizin aufgrund einer ausgeprägten Einschlafneigung am Tage (Epworth-Schläfrigkeits-Skala (ESS): 16 von 24 Punkten, $\mathrm{ab}>10$ Punkten gesteigerte Schläfrigkeit) vorgestellt hat. Als Vorerkrankung war ein chronisches Schmerzsyndrom bei rezidivierenden Bandscheibenvorfällen im LWS-Bereich bekannt. Die Dauermedikation bestand u.a. aus einer intrathekalen Opiatpumpentherapie (Dosierung: $230 \mathrm{mg} /$ die Morphinäquivalent). In der nächtlichen Polysomnografie zeigten sich ausgeprägte Atmungsstörungen, die ein periodisches Atemmuster aufwiesen ( Abb. 1). Der Apnoe-Hypopnoe-Index (AHI) betrug 43,4/h mit überwiegend zentralem Anteil (obstruktive Apnoen (OA): 2, zentrale Apnoen (ZA): 205, gemischte Apnoen (GA): 36). In der Auswertung der Ganznachtpolysomnografie fand sich ein erniedrigter REM- sowie Tiefschlafanteil mit 19,7\% bzw. 22,7\% der Gesamtschlafzeit (Total Sleep Time; TST). Die Einleitung einer nächtlichen Ventilationstherapie mittels nBIPAP-device im Bi-
Level-Modus mit Drücken von $16 / 7 \mathrm{~cm} \mathrm{H}_{2} \mathrm{O}$ im T-Modus führte zu einer weitgehenden Reduktion der Atmungsstörungen (AHI 3,3/h). Dementsprechend besserte sich auch die Schlafqualität, was sich am deutlichsten in einem Anstieg des Tiefschlafanteils auf 36,9\% der TST zeigte. Bei einer 3 Monate später stattgefundenen Therapiekontrolle berichtete der Patient von einer signifikanten Verbesserung des Tagesbefindens (ESS 11/24).

Fall 2: Vergleichbar präsentierte sich eine zweite 59-jährige Patientin (Gewicht $74 \mathrm{~kg}$, Größe $175 \mathrm{~cm}$, BMI 24,2 kg/m²), die bei ihrer Vorstellung ebenfalls eine sehr ausgeprägte Hypersomnie am Tage (ESS: 23/24) als Kardinalsymptom zeigte. Im Rahmen einer multisegmentalen Spondylodese bei Spondylolisthesis zehn Jahre zuvor wurde eine intrathekale Opiatpumpentherapie eingeleitet (Dosierung: 28,5 mg/die Morphinhydrochlorid). Auch hier ließen sich polysomnografisch deutliche Atmungsstörungen nachweisen. Der AHI in der diagnostischen Messung betrug 58,6/ h. (OA: 2, ZA: 34, GA: 228 s.o.). Im Schlafprofil zeigte sich ein deutlich erniedrigter REM- und leicht erniedrigter Tiefschlafanteil (6,4\%/TST bzw. 20,9\%/TST). Im Gegensatz zum ersten Fall war eine gewisse Regelmäßigkeit der Atmungstätigkeit erkennbar ( Abb. 2). In der Polysomnografie unter Ventilationstherapie (s.o. BiLevel, T-Mode, Beatmungsdruck: $14 / 6 \mathrm{~cm} \mathrm{H}_{2} \mathrm{O}$, Atemfrequenz: 12/min) zeigte sich eine Abnahme des AHI auf $0,7 / \mathrm{h}$ bei einer REM-Fraktion von $8,5 \%$ und einem Tiefschlafanteil von $10,8 \%$, bezogen auf die TST ( $\bullet$ Abb. 3). In der 3-Monatskontrolle wurde ein deutlicher Benefit unter Ventilationstherapie mit Verbesserung der Tagesschläfrigkeit (ESS 15/24) angegeben.

Fall 3: Beim dritten Patienten handelt es sich um einen 26-jährigen Mann (Gewicht 113 kg, Größe 184 cm, BMI 33,4 kg/m²) mit vorbekanntem Cauda-Equina-Syndrom. Der Patient beklagte ebenfalls eine ausgeprägte Einschlafneigung am Tage (ESS 18/ 24), Die analgetische Medikation umfasste Fentanylpflaster (100 $\mu$ g, alle drei Tage) sowie orales Morphinsulfat in unbekannter Dosierung bei teils exzessiver Eigenmedikation. Ein Aus- 


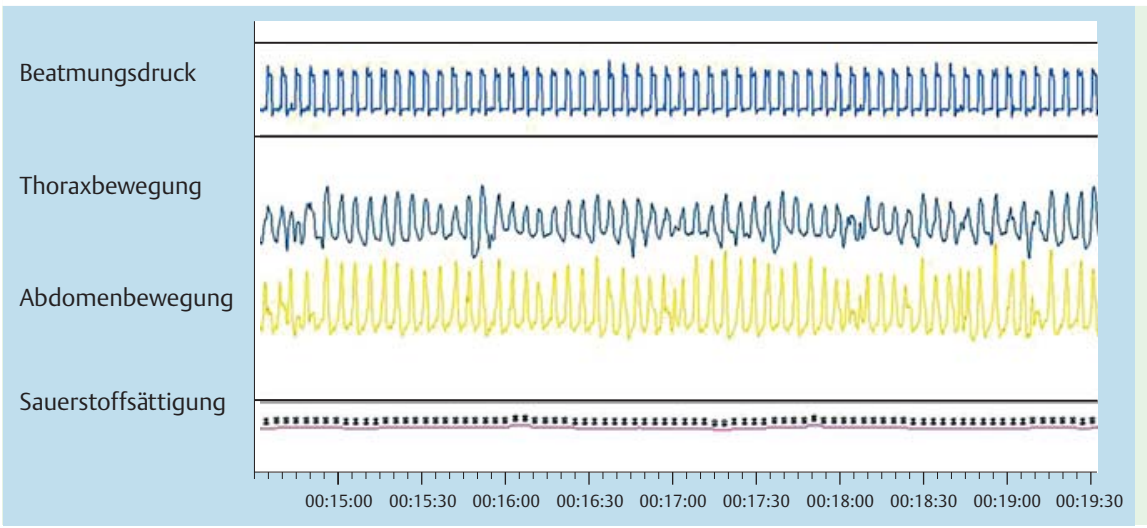

Abb. 3 Patient Nr. 2; 5-Minuten-Segment der Atmungsparameter aus der diagnostischen Polysomnografie unter Ventilationstherapie.

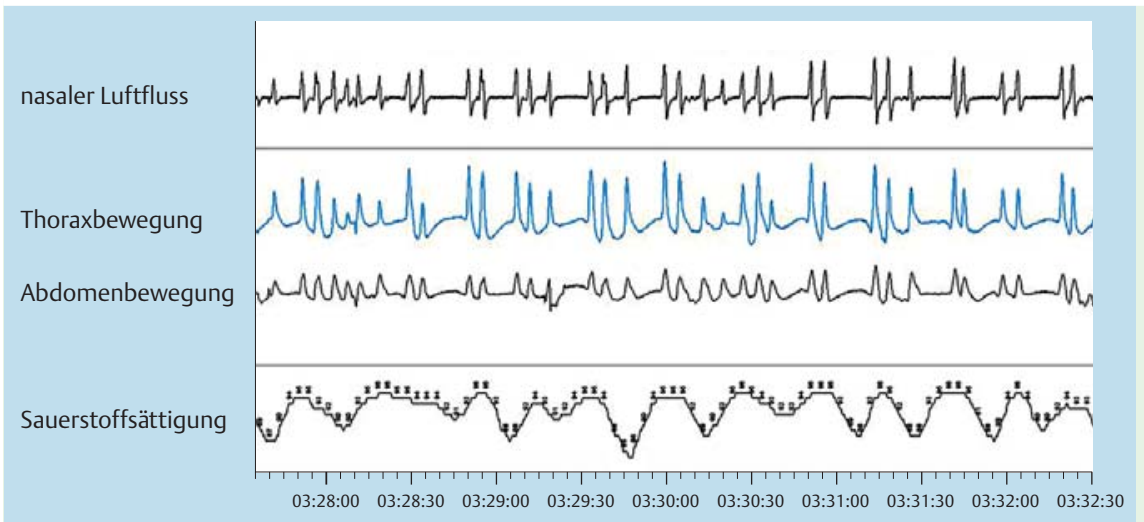

Abb. 4 Patient 3; 5-Minuten-Segment der Atmungsparameter aus der diagnostischen Polysomnografie. schnitt der Polysomnografie ist in $\bullet$ Abb. 4 dargestellt. Der AHI betrug 41,9/h (OA: 0, ZA: 176, GA: 27). Auffällig war hier der komplett fehlende REM-Schlaf. Eine nächtliche Beatmungstherapie (BiLevel, Beatmungsdruck: $16 / 8 \mathrm{~cm} \mathrm{H}_{2} \mathrm{O}$, T-Mode) reduzierte den AHI auf 6,6/h ohne wesentliche Veränderung des Schlafprofils. 12 Wochen nach Therapiebeginn beschreibt der Patient eine geringfügige Verbesserung der Hypersomnie (ESS 16/24).

\section{Diskussion}

Auch für chronische nicht tumorbedingte Schmerzpatienten gehört die Opioidtherapie heutzutage zum medikamentösen Standard. Schmerzpatienten wachen nachts oft auf und leiden demzufolge unter einer eingeschränkten Schlafqualität, die wiederum zu reduzierter Tagesbefindlichkeit und verstärkter Tagesmüdigkeit führen kann. Guter Schlaf ist ein wichtiges Kriterium für eine effiziente Schmerztherapie. Dass es bei Patienten mit einer chronischen Opioidtherapie jedoch auch zu einer medikamentös bedingten Störung des Schlafes kommen kann, sollte anhand der Fallberichte verdeutlicht werden.

In der S3-Leitlinie „Nicht erholsamer Schlaf/Schlafstörungen“ der deutschen Gesellschaft für Schlafforschung und Schlafmedizin sowie der „International Classification of Sleep Disorders (ISCD-2)“ der American Academy of Sleep Medicine werden Einteilungen der Schlafstörungen in unterschiedliche Kategorien vorgenommen $[22,23]$. Die Untergruppe der schlafbezogenen Atmungsstörungen (SBAS) gliedert sich in solche obstruktiver, zentraler, hypoventilationsbedingter, hypoxämischer oder anderer Genese. Die durch Opioidtherapie induzierten Atmungsstörungen sind der Gruppe der zentral bedingten Atmungsstörungen durch Medikamente, Drogen und andere Substanzen zuzuordnen.
Bei zwei der drei vorgestellten Patienten wurde eine intrathekale Schmerztherapie durchgeführt, bei dem dritten Patienten eine transdermale. Hinsichtlich der Effizienz sowie der Komplikationsrate unterschiedlicher Applikationsweisen der Opioidtherapie soll und kann in dieser Arbeit nicht explizit Stellung genommen werden.

Der Literatur zufolge sollte die Indikation zur intrathekalen Opioidtherapie bei nicht tumorbedingter Schmerztherapie jedoch kritisch geprüft werden, zumal die spinal applizierten Opioide eine engere therapeutische Breite aufweisen als orale und auch mit schwerwiegenderen Komplikationen zu rechnen ist [24-26]. Mangels prospektiver kontrollierter Studien bei Patienten mit nicht tumorbedingten Schmerzen ist ein valider Vergleich von Therapieeffizienz und Komplikationsrate von intrathekaler vs. transdermaler vs. oraler Opioidtherapie bislang nicht möglich. Gerade auch unter dem dargestellten Aspekt einer im Schlaf erhöhten Prävalenz zentral bedingter Atmungsstörungen sollte die individuelle Applikationsform und -dosierung kritisch hinterfragt werden.

Es ist weiterhin unklar, inwieweit schlafbezogene zentrale Atmungsstörungen bei Patienten mit nicht tumorbedingter Schmerztherapie zu einer relevanten Beeinträchtigung der Tagesbefindlichkeit sowie der Einschränkung der psychomentalen Leistungsfähigkeit führen. Der Sachverhalt, dass es bei unseren Patienten unter der eingeleiteten nicht invasiven Beatmung im Therapieverlauf zu einer Verbesserung der Tagesmüdigkeit gekommen ist, legt einen kausalen Zusammenhang zwischen Eliminierung der Atmungsstörungen und Verbesserung der Schlafqualität zwar nahe, beweist ihn aber nicht. In weitergehenden Untersuchungen mit entsprechend großen Patientenkollektiven sollte dieser Kausalzusammenhang spezifischer analysiert werden. Hier sollten über die ESS hinaus weitere Parameter zur 
Objektivierung der Tagesbefindlichkeit erhoben werden. Besonderes Augenmerk ist auch auf die mitunter gefährlichen Effekte der Kombinationsbehandlungen von Sedativa und Antidepressiva mit Opiaten zu richten. Nicht zuletzt bleibt die Frage abklärungsbedürftig, ob bei Patienten mit zentralen Atmungsstörungen im Vergleich zu Patienten ohne Atmungsstörungen auch eine erhöhte Morbidität und Mortalität besteht.

\section{Perspektive}

Zusammenfassend scheint es uns angesichts der zunehmenden Bedeutung der Opioidtherapie bei Patienten mit chronischen Schmerzzuständen dringend geboten, Schlaf und Atmung der betroffenen Patienten an einem ausreichend großen Kollektiv systematisch zu untersuchen. Patienten mit relevanten Atmungsstörungen sollten einer nichtinvasiven Beatmungstherapie zugeführt werden, insofern keine Veränderung der Dosierung sowie der Applikationsform des Opioids möglich ist.

\section{Interessenkonflikte}

Die Autoren geben an, dass kein Interessenkonflikt besteht.

\section{Literatur}

1 Wang D, Teichtahl H, Drummer $\mathrm{OH}$ et al. Central sleep apnea in stable methadone maintenance treatment patients. Chest 2005; 128: 1348 1356

2 Pattinson KTS. Opioids and the control of respiration. Br J Anaesth 2008; 100: $747-758$

3 Goldstein A, Lowney LI, Pal BK. Stereospecific and nonspecific interactions of the morphine congener levorphanol in the sucelluar fractions of mouse brain. Proc Natl Acad Sci USA 1971; 68: 1742 - 1747

4 Pert CB, Snyder SH. Opiate receptor: demonstration in nervous tissue. Science 1973; 179: 1011 - 1014

5 Lamotte C, Pert CB, Snyder SH. Opiate receptor binding in primate spinal cord: distribution and changes after dorsal root section. Brain Res 1976; 112: $407-412$

6 Yaksh TL, Rudy TA. Analgesia mediated by a direct spinal action of narcotics. Science 1976; 192: $1357-1358$

7 Wang JK, Nauss LA, Thomas JE. Pain relief by intrathecally applied morphine in man. Anesthesiology 1979; 50: 149-151
8 Holtsmann M, Fishman SM. Opioid receptors. In: Benton HT, Raja N, Molloy RE, Liu S, Fishman SM Essentials of Pain Medicine and Regional Anestesia. London: Churchill Livingstone, 2005: 87-91

9 Aghajanian GK. Tolerance of locus coeruleus neurones of morphine and suppression of withdrawal response by clonidine. Nature 1978; 276: $186-188$

10 Wilson L, Dorosz L. Possible Role of the opioid peptides in sleep. Med Hypotheses 1984; 14: 269-280

11 Kay DC, Pickworth WB, Neidert GL et al. Opioid effects on computer-derived sleep and EEG parameters in nondependent human addicts. Sleep 1979; 2: 175 - 191

12 Kay DC, Pickworth WB, Neider GL. Morphine-like insomnia from heroin in nondependent human addicts. Br J Clin Pharmacol 1981; 11: 159 169

13 Pickworth WB, Neidert GL, Kay DC. Morphinelike arousal by methadone during sleep. Clin Pharmacol Ther 1981; 30: 796-804

14 Dimsdale JE, Norman D, DeJardin D et al. The effects of opioids on sleep architecture. JCSM 2007; 3: 33-36

15 Donald KW, Christie RV. The respiratory response to carbon dioxide and anoxia in emphysema. Clin Sc 1949; 8: 33-44

16 Wilson RH, Hoseth W, Dempsey ME. Respiratory Acidosis - I. Effects of Decreasing Respiratory Minute Volume in Patients with Severe Chronic Pulmonary Emphysema, with Specific Reference to Oxygen, Morphine and Barbiturates. Am J Med 1954; 17: 464-470

17 Teichtahl H, Wang D. Sleep-disordered breathing with chronic opioid use. Expert Opin Drug Saf 2007; 6: 641-649

18 Farney RJ, Walker JM, Cloward TV et al. Sleep-disordered breathing associated with long-term opioid therapy. Chest 2003; 123: 632-639

19 Wang $D$, Teichtahl $H$. Opioids, sleep architecture and sleep-disordered breathing. Sleep Med Reviews 2007; 11: $35-46$

20 Walker JM, Farney RJ, Rhondeau SM et al. Chronic opioid use is a risk factor for the development of central sleep apnea and ataxic breathing. J Clin Sleep Med 2007; 3: 455 - 461

21 Ko S, Goldstein DH, VanDenKerkhof EG. Definitions of „respiratory depression“" with intrathecal morphine postoperative analgesia: a review of the literature. Can J Anesth 2003; 50: 679-688

22 American Academy of Sleep medicine2005. International classification of sleep disorders. Diagnostic and coding manual; 2nd ed, Westchester, Illinois: American Academy of Sleep Medicine, 2005

23 Mayer G, Fietze I, Fischer J et al. S3-Leitlinie Nicht-erholsamer Schlaf/ Schlafstörungen. Somnologie 2009; 13 (Suppl. 1): 4-160

24 Kindler D, Maier C, Kagel T et al. Neurologische Komplikationen und Wirkverlust unter intrathekaler Schmerztherapie. Schmerz 2005; 19: $144-155$

25 Kumar K, Hunter G, Demeria DD. Treatment of chronic pain by using intrathecal drug therapy compared with conventional pain therapies: a cost-effectiveness analysis. J Neurosurg 2002; 97: 803-810

26 Xiulu $R$. Drug related side effects of long-term intrathecal morphine therapy. Pain Physician 2007; 10: 357-365 